Article

\title{
Predictors of Adrenal and Gonadal Hormones in Relation to Biological and Management Factors Among Captive Red Pandas in Indian Zoos
}

\author{
Aamer Sohel Khan'1, Janine Brown², Vinod Kumar³, Govindswamy Umapathy³, and Nagarajan Baskaran1,*
}

1Dept. of Zoology and Wildlife Biology, A.V.C College (Autonomous), Mannampandal, Mayiladuthurai - 609305, Tamil Nadu, India;khannaamirsohel@gmail.com, nagarajan.baskaran@gmail.com

${ }^{2}$ Smithsonian Conservation Biology Institute, Center for Species Survival, MRC 55331500 Remount Road Royal, VA 22630; BrownJan@si.edu

${ }^{3}$ Laboratory for the Conservation of Endangered Species, CSIR-Center for Cellular and Molecular Biology, Uppal Road, Hapsiguda 500007, Hyderabad, Telangana, India; vinod@ccmb.res.in, guma@ccmb.res.in

* Correspondence: nagarajan.baskaran@gmail.com; Tel.: + 91-4364-8903410794 (Mobile)

Simple Summary: Red pandas are a threatened species and zoos worldwide are working to conserve the species through international captive breeding programs. However, information on the physiology of captive red pandas is lacking, which is hampering efforts to increase reproductive success and meet captive breeding goals. This study evaluated adrenal and reproductive hormones in relation to environment and biological factors to identify predictors of adrenal stress and gonadal hormones among male and female red pandas housed in three Indian zoos. Data reveal that fecal glucocorticoid metabolite concentrations in red pandas were influenced by sex, visitor numbers, frequency of feedings, and enclosure area. Concomitantly, fecal androgen metabolites in males and fecal progestagen metabolites in females were influenced by age, tree density in the enclosure, visitor numbers, and frequency of feedings. This study suggests housing animals in larger enclosures with more trees, employing more frequent feeding schedules, and controlling the number of visitors all can have positive effects on welfare of red pandas.

\begin{abstract}
Animals in human care are affected by stressors that can ultimately reduce fitness. When reproduction is affected, endangered species' conservation programmes can be severely compromised. Thus, understanding factors related to stress and reproduction, and measures of related hormones, are important to ensure captive breeding success. Red pandas are endangered and populations in the wild are threatened with extinction. A global captive breeding programme has been launched to conserve the species with the goal of reintroduction. However there is little informaiton on how stressors impact reproductive aspects of the species. This study measured fecal glucocorticoid (fGCM), fecal progestagen (fPM) and fecal androgen (fAM) metabolite concentrations in 12 female and 8 male red pandas (Ailurus fulgens fulgens) at three zoos in northeastern India to determine predictors of adrenal and gonadal steroid activity and the influence of fGCM on reproduction. Results indicated that fGCM concentrations were higher in males than females, and positively correlated with number of visitors, while negatively related to frequency of feedings and enclosure area. Sex, visitor number, frequency of feeding, and enclosure area explained $67 \%$ of the variations in fGCM concentrations in the study population. Concentrations of fPM were positively associated with tree density in the enclosure, explaining $47 \%$ of the variation among females. For fAM, positive associations were found with frequency of feeding, but concentrations were negatively related to age and number of visitors; these three covariates explained $45 \%$ of the variation in fAM concentration among males. Comparison of fGCM with fPM showed a negative trend, indicating
\end{abstract}


increasing adrenal hormones may decrease reproductive function among female red pandas. The study thus suggests that zoo management should consider increasing feeding frequency, providing larger enclosures with more trees, and regulating visitor numbers to reduce stress and increase reproductive fitness among red pandas.

Keywords: Captive breeding; endangered, red panda, reproductive hormone; stress hormone, welfare

\section{Introduction}

The red panda (Ailurus fulgens) is an endemic species to the eastern Himalayas from Nepal, India, Bhutan, China extending up to Myanmar, and lives at altitudes of $1500-4000 \mathrm{~m}$ [1-3]. The species is habitually nocturnal and belongs to a monophyletic family - Ailuridae, which consists of two sub-species, Ailurus fulgens fulgens and Ailurus fulgens styani [1]. Red pandas currently are threatened throughout much of their ranges[2] due to poaching and destruction of bamboo forests [1,4]. The wild population is on a declining trend with less than 10,000 mature individuals remaining [2]. Worldwide efforts to protect the species include global captive breeding programmes with the goal of reintroduction $[2,5,6]$. Nevertheless, populations are not stable in captivity either [3,7], owing to low reproductive success and poor infant survival [7].

Worldwide there are 92 institutions that house more than 300 red pandas [2], with a total of 36 individuals in India. Studbook data suggests that the breeding success of red pandas across institutions varies considerably [8]. Environmental factors and general husbandry practices can exert stress in captive animals [9], although responses often are species-specific [10]. Environmental and biological factors such as presence of visitors, enclosure size, diet, enrichment, age, sex can affect stress responses in numerous species [9,11-13]. In red pandas, it has been shown that high ambient temperatures, small enclosures, improper enclosure substrates, lack of hiding places, next boxes and climbing structures, and exposure to visitors all may contribute to maternal stress and reduced reproductive success in captivity $[5,8,14]$. Climate factors, in particular, maybe be a factor as behaviour is compromised by warmer weather in places where many captive populations are kept [2,5,8]. Offspring survival is higher in younger as compared to older pandas [15], indicating age may also influence breeding success.

A few studies have evaluated glucocorticoids and/or progestagens in captive red pandas [7,16-18], but overall the reproductive physiology of this species is poorly understood, especially with regards to the effects of management factors. This study investigated how environmental and biological factors were related to stress and reproductive hormones in Ailurus fulgens fulgens, and how those in turn were related to reproduction. Fecal glucocorticoid (fGCM), androgen (fAM) and progestogen (fPM) metabolite concentrations were assessed in male and female pandas at three zoos in northeastern India. Our hypotheses were that i) variations in environmental and biological factors influence fGCM, fAM and fPM concentrations, and ii) fGCM concentrations are negatively related to fAM and FPM concentrations. Because captivity is known to influence the fitness of individuals, understanding factors related to stress and reproduction, and measures of related hormones, is important to ensure captive breeding success.

\section{Materials and Methods}

Study Sites

This study was carried out in three zoological facilities in India: Padmaja Naidu Himalayan Zoological 
Park (PNHZP), Darjeeling; 2) Sikkim Himalayan Zoological Park (SHZP), Gangtok; and 3) G. B. Pant High Altitude Zoo (GBPHAZ), Nainital. All zoos participate in the Red Panda Global Species Management Plan. The first captive breeding center in the country for the species was established at PNHZP in 1957. The enclosures mimick a wet temperate forest climate with temperature variation from sub-zero in winter to $25^{\circ} \mathrm{C}$ during the summer. Animals in this study were maintained in semi natural open enclosures with large trees and nests. Bamboo was fed in the evening with supplementary diet items (honey, milk, egg, and fruits) in the morning. SHZP housed individuals in large open enclosures with only a few trees and nests. The diet consisted of bamboo in the evening and supplementary foods (honey, milk, egg, fruits, and breads) in the morning. The temperature varies between $4-22^{\circ} \mathrm{C}$. GBPHAZ kept individuals in small netted enclosures with a nest box, but no trees. Bamboo and supplementary diet items (honey, milk, egg, and fruits) were fed together in the morning. The temperature at GBPHAZ fluctuates from 3 to $25^{\circ} \mathrm{C}$. All three zoos were at a similar altitude. Breeding records for each female were obtained by the registar at each zoo. Pandas were managed either as solitary or in male-female pairs during the breeding season (January - March). Females with young cubs were also housed together at zoos (see Supplementary Table 1). The four biological and nine environmental covariates assessed in the study are defined in Table 1. 
Table 1: Details of biological and environmental covariates collected during study.

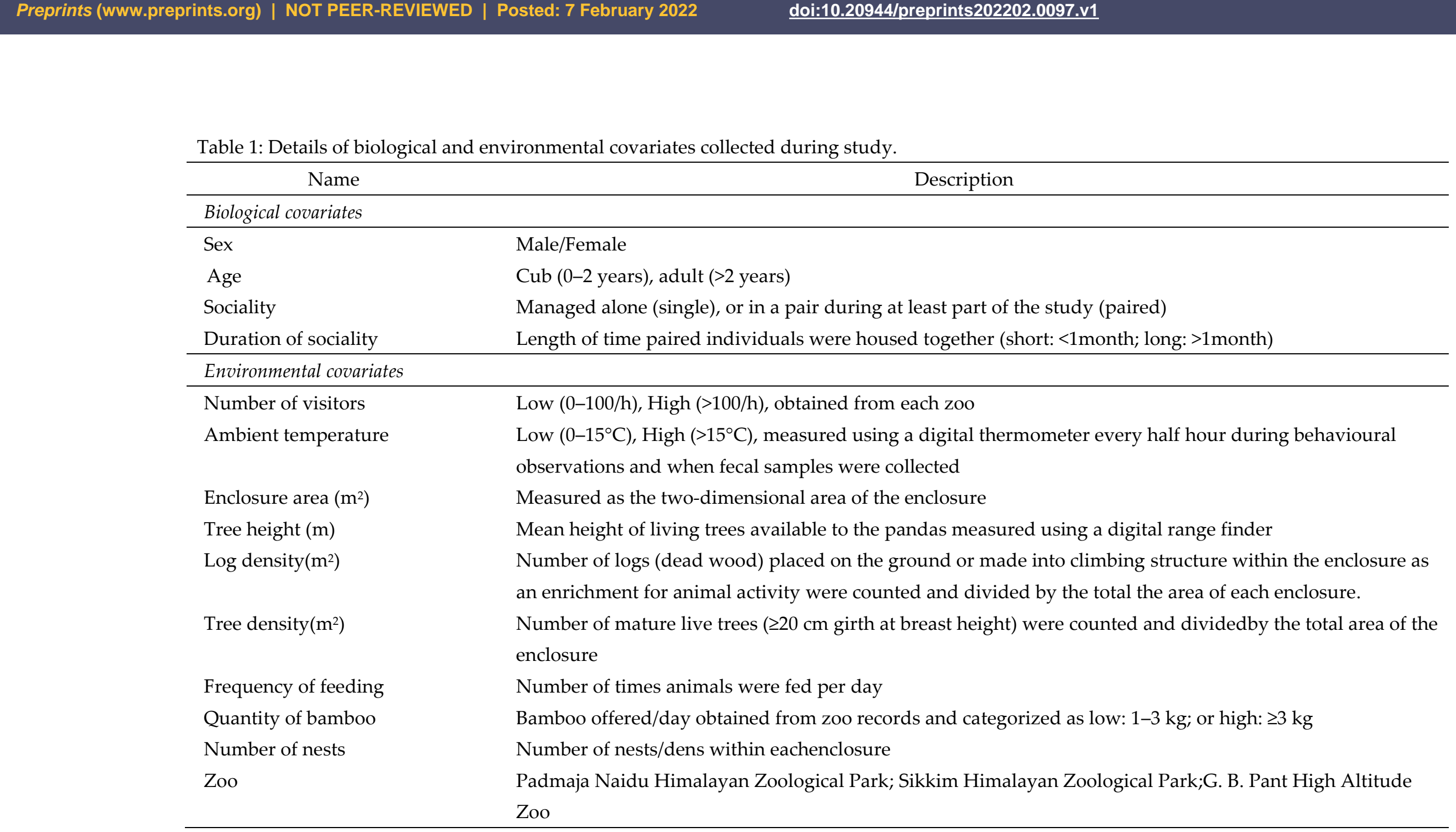

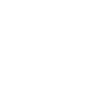

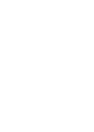


Fecal sample collection

A total of 40 fecal samples were collected from 20 red pandas (12 females and 8 males) (Supplementary Table 1), with two samples from each individual at a 2-week interval between December 2017 and February 2018. Entire fresh fecal samples (excluding the outer shiny layer) were collected in the morning [18]. Individual red pandas defecate at the same place in the enclosure, which aided individual identification. Immediately after collection, the samples were dried at $70^{\circ} \mathrm{C}$ for 24 hours in a hot air oven [19]. The dried samples were packed in sterile zip-lock bags and stored at room temperature in a moisture-controlled room. Samples were transferred within 2 weeks to the Laboratory for the Conservation of Endangered Species (LaCONES), CCMB Hyderabad, India, for processing and hormonal analyses.

Extraction of fecal steroid metabolites

Fecal steroid metabolites were extracted using protocols described earlier [18,20,21]. Approximately, $0.2 \mathrm{~g}$ of dried fecal powder was boiled in $5 \mathrm{ml}$ of $90 \%$ ethanol for $20 \mathrm{~min}$. The samples were centrifuged at $500 \mathrm{~g}$ for $20 \mathrm{~min}$ and supernatant transferred to a fresh tube. The pellets were re-suspended in $5 \mathrm{ml}$ of $90 \%$ ethanol, vortexed for $1 \mathrm{~min}$, recentrifuged and both supernatants combined. The supernatants were dried at $40 \mathrm{C}$ in an oven, re-suspended in $1 \mathrm{ml}$ of absolute methanol and vortexed for $1 \mathrm{~min}$. Fecal extracts were kept at -20 until analyzed by enzymeimmunoassay (EIA).

Enzyme immunoassays

fGCM were quantified using a polyclonal cortisol antibody (R4866, Coralie Munro, University of California, Davis), which had been validated for use in red panda feces $[18,22]$. The sensitivity of the assay was $1.95 \mathrm{pg} /$ well. The inter and intra-assay coefficients of variation (CV) were $7.9 \%$ and $5.5 \%$, respectively.

fPM concentrations were quantified using a $5 \alpha$-pregnan-3 $\alpha$-ol-20-one EIA (polyclonal antibody) validated for red panda feces $[18,21,23]$. The inter and intra-assay coefficients of variation were $9.4 \%$ and $7.3 \%$, respectively.

fAM concentrations were quantified using a polyclonal testosterone antibody (R156/7; Coralie Munro, University of California, Davis), validated for measuring fecal androgens in red panda [18] following protocols described previously $[18,22]$. The inter and intra-assay coefficient of variation (CV) were $8.2 \%$ and $6.1 \%$, respectively.

\section{Statistical analyses}

Data on dependent factors (fGCM, fPM and fAM) were first tested for normality. The fGCM and fPM data were not normal and could not be normalized by any of four transformations, and so were tested using Mann-Whitney U tests. The fAM data were normally distributed (Kolmogorov-Smirnov test, $p=0.200$ and Shapiro-Wilk test, $p=0.450$ ) and were evaluated using analysis of variance (ANOVA). To determine potential predictors of stress and reproductive hormones, we used a Regression with Empirical Variable Selection (REVS) model in LEAPS package with all 14 covariates listed in Table 1. In REVS all-subset regression is run on data set using LEAPS, this quanitfy emperical support for each independent varaibles. Several models were created, first with most empirical support, second with the first variable and the second most empirical support and so on. The best model was determined by comparing $\mathrm{R}^{2}$ and delta AIC values; the model with the higher $\mathrm{R}^{2}$ and lower delta AIC was selected [24]. 
To explore the effect of stress on reproduction, linear regression was used with fGCM as the independent and fPM and fAM as dependent variables. Further, to control for the interference/componding effects of potential covariates (ambient temperature, number of visitors, tree density, log density and number of nests) on the relationship between fGCM and fPM, analysis of co-variance (ANCOVA) was used. All analyses were conducted using SPSS (SPSS Inc., Version 16) and R (Version 13.4.1).

\section{Results}

Mean fGCM concentration was $44.00 \pm 5.16 \mathrm{ng} / \mathrm{g}$ (range: 2.84 to $198.91 \mathrm{ng} / \mathrm{g} ; n=40$ ), with males having higher concentrations $(49.19 \pm 11.12 \mathrm{ng} / \mathrm{g}, n=16)$ than females $(40.56 \pm 4.49 \mathrm{ng} / \mathrm{g}, n=24 ; \mathrm{U}=185, p<0.05)$. Visitor number positively influenced fGCM concentrations $\left(\mathrm{R}^{2}=0.60 ; F=56.5, p=4.972 \mathrm{e}-9 ; p<0.05\right)($ Table 2 , Fig. 1). By contrast, fGCM concentrations in red pandas housed in enclosures with taller trees were lower compared to those with shorter trees $(p<0.05)$. Similarly, fGCM concentrations were higher among pandas manged in smaller than larger enclosures $(p<0.05)$, and also with a low versus high frequency of feedings $(p<0.05)$.

Table 2: Mean ( \pm SE) concentrations of adrenal and reproductive hormones in relation to independent biological and environmental factors among captive red pandas in India, $\mathrm{U}=$ Mann-Whitney $\mathrm{U}$ test, $\mathrm{H}=$ Kruskal Wallis test, $\mathrm{F}=$ analysis of variance, $n=$ sample size in category. Stastical test results with significant levels are bolded.

\begin{tabular}{|c|c|c|c|c|}
\hline Covariate & Category & $\begin{array}{l}\text { fGCM } \\
\text { (ng/g) }\end{array}$ & $\begin{array}{c}\mathrm{fPM} \\
(\mu \mathrm{g} / \mathrm{g})\end{array}$ & $\begin{array}{c}\text { fAM } \\
\text { (ng/g) }\end{array}$ \\
\hline Biological & & (n) & (n) & (n) \\
\hline \multirow[t]{3}{*}{ Age } & Adult & $42.31 \pm 5.727(17)$ & $3.12 \pm 2.48(10)$ & $15.87 \pm 2.320(7)$ \\
\hline & $\mathrm{Cub}$ & $53.63 \pm 11.70(3)$ & - & - \\
\hline & Test ( $p$ value) & $\mathrm{U}=68.5(0.204)$ & - & - \\
\hline \multirow[t]{3}{*}{ Sociality } & Paired & $45.24 \pm 5.351(19)$ & $2.63 \pm 2.07(10)$ & $16.82 \pm 2.62(6)$ \\
\hline & Solitary & $20.67 \pm 8.955(1)$ & - & $10.22 \pm 0.76(1)$ \\
\hline & Test ( $p$ value) & $\mathrm{U}=68(0.204)$ & - & $\mathrm{F}=4(0.170)$ \\
\hline \multirow[t]{3}{*}{ Duration of sociality } & Short & $47.18 \pm 6.187(16)$ & $3.83 \pm 3.09(7)$ & $14.13 \pm 2.29(5)$ \\
\hline & Long & $31.37 \pm 5.974(4)$ & $0.28 \pm 0.05(3)$ & $23.52 \pm 4.78(2)$ \\
\hline & Test ( $p$ value) & $\mathrm{U}=85.5(0.150)$ & $\mathrm{U}=24(0.449)$ & $F=6(0.030)$ \\
\hline \multicolumn{5}{|l|}{ Environmental } \\
\hline \multirow[t]{3}{*}{ Ambient temperature } & Low & $40.38 \pm 3.637(18)$ & $3.45 \pm 2.75(9)$ & $17.33 \pm 2.39(7)$ \\
\hline & High & $76.66 \pm 40.956(2)$ & $0.16 \pm 0.07(1)$ & $7.07 \pm 4.46(1)$ \\
\hline & Test ( $p$ value) & $\mathrm{U}=58.5(0.543)$ & $\mathrm{U}=7(0.165)$ & $F=0.927(0.355)$ \\
\hline \multirow[t]{3}{*}{ Number of visitors } & Low & $35.90 \pm 3.020(17)$ & $3.60 \pm 2.91(8)$ & $18.46 \pm 2.32(5)$ \\
\hline & High & $89.98 \pm 23.396(2)$ & $0.42 \pm 0.20(2)$ & $6.37 \pm 2.66(2)$ \\
\hline & Test ( $p$ value) & $\mathrm{U}=21.5(0.002)$ & $\mathrm{U}=20.5(0.586)$ & $F=4.95(0.046)$ \\
\hline \multirow[t]{3}{*}{ Tree height (m) } & Low & $50.81 \pm 7.081(13)$ & $0.79 \pm 0.21(7)$ & $13.46 \pm 2.54(4)$ \\
\hline & High & $29.90 \pm 4.023(7)$ & $8.57 \pm 8.29(3)$ & $19.08 \pm 4.128(3)$ \\
\hline & Test ( $p$ value) & $U=99(0.027)$ & $\mathrm{U}=37(0.680)$ & $\mathrm{F}=3(0.108)$ \\
\hline Enclosure area $\left(\mathrm{m}^{2}\right)$ & Small & $62.1 \pm 14.233(6)$ & $13.08 \pm 1.23(2)$ & $12.76 \pm 3.764(2)$ \\
\hline
\end{tabular}




\begin{tabular}{|c|c|c|c|c|}
\hline & Large & $36.28 \pm 3.495(14)$ & $0.63 \pm 0.16(8)$ & $17.11 \pm 2.898(5)$ \\
\hline & Test ( $p$ value) & $\mathrm{U}=106.5(0.050)$ & $\mathrm{U}=32(0.037)$ & $F=4.61(0.053)$ \\
\hline \multirow[t]{3}{*}{ Tree density } & Low & $44.66 \pm 5.556(18)$ & $0.571 \pm 0.151(9)$ & $17.34 \pm 2.398(6)$ \\
\hline & High & $36 \pm 6.300(2)$ & $27.00 \pm 23.95(1)$ & $7.07 \pm 4.460(1)$ \\
\hline & Test ( $p$ value) & $\mathrm{U}=49.5(0.758)$ & $\mathrm{U}=1(0.032)$ & $F=2.04(0.178)$ \\
\hline \multirow[t]{3}{*}{ Log density } & Low & $44.11 \pm 6.758(15)$ & $0.58 \pm 0.18(6)$ & $17.11 \pm 2.898(5)$ \\
\hline & High & $43.75 \pm 6.382(5)$ & $9.05 \pm 8.21(4)$ & $12.77 \pm 3.764(2)$ \\
\hline & Test ( $p$ value) & $U=132.5(0.413)$ & $\mathrm{U}=33(0.458)$ & $F=3.14(0.101)$ \\
\hline \multirow[t]{3}{*}{ Number of nests } & Low & $44.41 \pm 7.024(13)$ & $0.49 \pm 0.18(6)$ & $17.04 \pm 2.779(5)$ \\
\hline & High & $43.19 \pm 6.660(7)$ & $9.27 \pm 8.16(4)$ & $12.94 \pm 4.440(2)$ \\
\hline & Test ( $p$ value) & $\mathrm{U}=160.5(0.665)$ & $\mathrm{U}=11.5(\mathbf{0 . 0 1 2})$ & $\mathrm{F}=0.38(0.550)$ \\
\hline \multirow[t]{3}{*}{ Frequency of feeding } & Low & $54.56 \pm 9.002(10)$ & $0.90 \pm 0.29(4)$ & $11.40 \pm 2.308(4)$ \\
\hline & High & $33.46 \pm 4.079(10)$ & $4.61 \pm 4.13(6)$ & $21.83 \pm 3.208(3)$ \\
\hline & Test ( $p$ value) & $\mathrm{U}=129.5(0.050)$ & $\mathrm{U}=34(0.280)$ & $F=7.35(0.019)$ \\
\hline \multirow[t]{3}{*}{ Quantity of bamboo } & Low & $65.83 \pm 20.034(4)$ & $0.57 \pm 0.25(2)$ & $7.07 \pm 4.460(1)$ \\
\hline & High & $38.56 \pm 3.800(16)$ & $3.76 \pm 3.09(8)$ & $17.33 \pm 2.398(6)$ \\
\hline & Test ( $p$ value) & $\mathrm{U}=86(0.155)$ & $\mathrm{U}=30(0.850)$ & $F=2.71(0.125)$ \\
\hline \multirow[t]{4}{*}{ Zoo } & Zoo1 & $40.31 \pm 3.786(17)$ & $0.62 \pm 0.16(8)$ & $17.34 \pm 2.400(6)$ \\
\hline & Zoo2 & $41.53 \pm 17.160(1)$ & $26.10 \pm 2.38(1)$ & - \\
\hline & Zoo3 & $76.77 \pm 40.956(2)$ & $0.16 \pm 0.071(1)$ & $7.07 \pm 4.460(1)$ \\
\hline & Test ( $p$ value) & $\mathrm{H}=0.37(0.831)$ & $\mathrm{H}=5.93(0.052)$ & $\mathrm{H}=2.70(0.100)$ \\
\hline
\end{tabular}

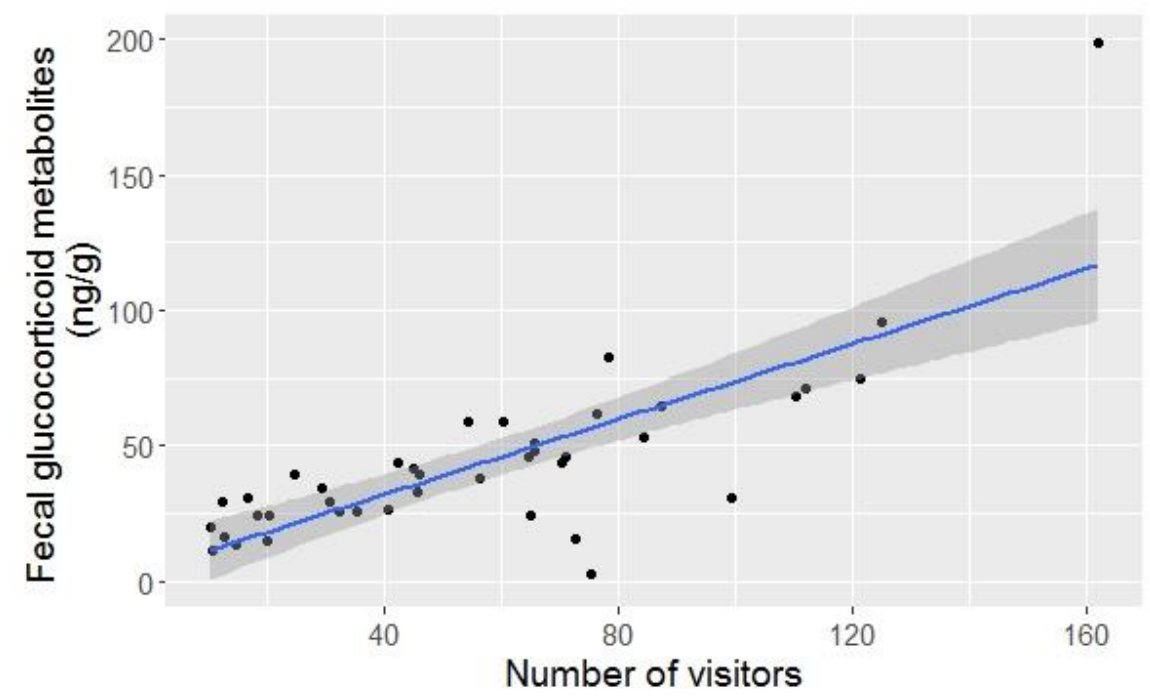

Fig. 1: Relationship between number of visitors and fecal glucocorticoid metabolite concentrations in red pandas at three Indian zoos.

Mean fPM concentration among adult females was $3.12 \pm 2.47 \mu \mathrm{g} / \mathrm{g}(n=12)$. Adults managed in enclosures with a higher tree density had higher fPM concentrations compared to those with a lower tree 
density $(p<0.05)$ and similarly with number of nests $(p<0.05)$ (Table 2$)$. Conversely, adult females managed in smaller enclosures showed higher fPM than those in larger enclosures $(p<0.05)$.

The fAM concentrations in adult males averaged 15.87 $\pm 2.32 \mathrm{ng} / \mathrm{g}(n=8)$. Concentrations were higher among individuals exposed to a lower compared to a higher visitor number $(p<0.05)$, and among individuals with a higher compared to a lower frequency of feedings $(p<0.05)$. Similarly, individuals managed in social situations for longer periods had higher concentrations of fAM compared to those with shorter durations, and the trend was the same i.e., positive with regards to enclosure size, although the data only approached significance $(p=0.053)$.

Among the 13 covariates tested against fGCM using the REVS model, concentrations decreased with increased frequency of feedings and enclosure area, but increased with number of visitors and males compared to females (Table 3). These four factors explained 67\% of the variations in fGCM concentrations in captive red pandas. In the case of $\mathrm{fPM}$, tree density with a positive trend was the only predictor, explaining $47 \%$ of the variation among the females sampled (Table 3). Conversely, the REVS model for fAM predicted that concentrations were increased significantly by feeding frequency and decreased with number of visitors and age of the individuals. These three covariates explained $45 \%$ of the variations in fAM concentration among male red pandas (Table 3).

Table 3: Regression with Empirical Variable Selection (REVS) model to explore the effect of biological and environmental covariates on physiology of captive red pandas at three Indian zoos.

\begin{tabular}{|c|c|c|c|c|c|c|c|}
\hline $\begin{array}{l}\text { Dependent } \\
\text { variable }\end{array}$ & Predictor covariate & Estimate $\pm \mathrm{SE}$ & $\mathrm{t}$ & $\operatorname{Pr}(>|t|)$ & $\begin{array}{c}\mathrm{AIC} \\
(\Delta \mathrm{AIC})\end{array}$ & $\mathrm{p}$ & Adj. $\mathrm{R}^{2}$ \\
\hline \multirow[t]{5}{*}{ fGCM } & (Intercept) & $3.91 \pm 14.635$ & 0.27 & 0.790 & 240 & $9.610 \mathrm{e}-9$ & 0.67 \\
\hline & Number of visitors & $0.75 \pm 0.087$ & 8.59 & $3.87 \mathrm{e}-10 * * *$ & $(0.00)$ & & \\
\hline & Frequency of feedings & $-19.85 \pm 7.287$ & -2.73 & $0.001 * *$ & & & \\
\hline & Sex & $13.17 \pm 6.276$ & 2.10 & $0.042 *$ & & & \\
\hline & Enclosure area & $-0.007 \pm 0.002$ & -2.07 & $0.046^{*}$ & & & \\
\hline \multirow[t]{2}{*}{ fPM } & (Intercept) & $-1559 \pm 1751$ & -0.89 & 0.382 & 429 & $0.126 \mathrm{e}-3$ & 0.47 \\
\hline & Tree density & $1080 \pm 232$ & 4.63 & $0.125 \mathrm{e}-3 * * *$ & $(0.00)$ & & \\
\hline \multirow[t]{4}{*}{ fAM } & (Intercept) & $-0.17 \pm 6.240$ & -0.02 & 0.984 & 64.5 & 0.018 & 0.45 \\
\hline & Frequency of feedings & $12.65 \pm 3.782$ & 3.33 & $0.006 * *$ & $(0.00)$ & & \\
\hline & Age & $-0.97 \pm 0.334$ & -2.90 & $0.012 *$ & & & \\
\hline & Number of visitors & $-0.10 \pm 0.050$ & 2.17 & $0.049 *$ & & & \\
\hline
\end{tabular}

Significance: $* * * 0.001, * * 0.01, * 0.05$.

Linear regressions with $\mathrm{fPM}$ as the dependent variable showed a decreasing trend with increasing fGCM concentrations, although it was not significant $\left(\beta=-12.93, R^{2}=0.001, p=0.895\right)$. However, when controlled for tree density using ANCOVA, the relationship was significant $(\beta=-4286, t=-4.65, p=1.56 \mathrm{e}-4 ; F$ $\left.(3,20)=217.6, p=1.97 \mathrm{e}-15, \eta p^{2}=0.55\right)$. There was no relationship between fGCM and fAM in male pandas $\left(\beta=-0.02, R^{2}=0.005, p=0.768\right)$. 
Based on breeding records at each facility (Supplementary Table 2), three out of eight pandas never gave birth, and the reproductive rate of all individuals was less than $50 \%$, indicating poor breeding rates among the captive red panda in India.

\section{Discussion}

This study found a number of biological and environmental factors affected gonadal and adrenal hormones in zoo-housed red pandas in India. The frequency of feeding, enclosure area, visitor number, and sex were significant predictors explaining $67 \%$ of the variations in the fGCM concentrations in males and females, whereas tree density within the enclosure was a positive predictor of fPM concentrations among female pandas, explaining $47 \%$ variation. In males, the frequency of feeding was positive, and visitor number and age were negative drivers of fAM concentrations, explaining $45 \%$ variation. Thus, housing red pandas in more natural enclosures that are larger and have taller trees, including enrichment, like climbing structures and nest boxes for hiding, and feeding bamboo more frequently are important factors to supporting good reproduction and welfare in captive red pandas.

Visitor intensity was a significant and positive predictor of fGCM concentration in this study, indicating an impact of high visitor numbers on physiological function. Visitors are known to have negative effects on animal welfare and are one of the potential stressors for animals in confinement [25]. The red panda is nocturnal by nature, so when continuously exposed to large numbers of visitors during the day, up to 162 per hour, it should not be surprising that increasing stress with visitor intensity is an outcome. Relationships between visitors and fGCM concentrations have been reported in a number of captive species [26], including Mexican wolves [27], Indian blackbuck [28], Royal Bengal tiger and Indian leopard [12].

The frequency of feeding was one of the key predictors of decreasing trends in fGCM concentrations, indicating the importance of an adequate food supply on the welfare of captive red pandas. A nutritious and balanced diet is essential for the survival of all organisms, and for animals in captivity, unlike wild cohorts, it is dependent on humans. Therefore, captive animals with an adequate food supply are expected to have less nutritional stress, as observed in the present study; i.e., decreased fGCM concentrations with frequency of feeding. The red panda has a relatively inefficient digestive ability to process bamboo, their principal food component, being able to extract only one quarter of the energy, necessitating that they spend a large amount of time foraging [29]. Thus, frequent feeding appears to be an essential parameter to reduce frustration and stress in captive red panda, as reported in other species [9]. Similar to the present findings, incorporating frequent feeding regimens was better for the welfare of zoo elephants [30], and reduced stereotypic behaviours, a psychological indicator of stress, in captive Asian elephants [31]. In free ranging African elephants, the relationship between rainfall, a proxy for food availability, and fGCM concentrations was negative [32]. Similarly, a study on chimpanzees showed a negative association between monthly fruit abundance (food availability) and urinary cortisol concentration [33]. Our results further strengthen earlier findings that a more constant food supply through frequent feedings can potentially support good welfare in captive animals.

This study identified sex as a significant predictor of fGCM, with male pandas having higher concentrations compared to females. The red panda is a species with a promiscuous polygynous mating system, where (i) males have larger body mass than females, (ii) males, unlike females, do not have equal chances of mating opportunities, as high quality males have higher reproductive success, (iii) males compete among themselves to mate with receptive females, and (iv) males range over a larger area compared to 
females [34]. Therefore, males with a polygynous mating system are expected to have higher adrenal glucocorticoid activity than females, which agrees with earlier studies on chimpanzees [33], and captive jungle cats [11].

The present study showed that enclosure size was associated negatively with fGCM concentrations, indicating an importance of adequate exhibit area on the welfare of this species. Enclosure size for any species in a zoo setting should take into consideration the home range size in the wild [35], which for red pandas is estimated to be $9.6 \mathrm{~km}^{2}[36,37]$. However, providing adequate enclosure areas is not always possible in zoos, especially in India, as they are located in densely populated cities, where space is a major constraint. Restricted movement in captivity is known to induce physiological stress in animals and has a great impact on their ability to exhibit naturalistic behaviours [9,38,39]. Glucocorticoid concentrations have been shown to decrease with increased enclosure size in big cats [40] and cheetahs [41], which agrees with our finding that red pandas in larger enclosures had lower fGCM concentrations.

Under natural conditions, the red panda is an arboreal species and well-known to use trees for day-to-day activities like feeding, resting, moving, including constructing nests for parturition and rearing offspring. The presence of trees could therefore be an important cue for normal physiological responses [42]. Free-ranging female elk during the summer and autumn, when habitat conditions are poor in terms of nutrition, exhibit lower progesterone concentrations that can potentially interrupt pregnancy [43]. Wild spider monkeys in disturbed habitats also exhibited higher cortisol concentrations than those in protected habitats [44]. Therefore, under free-ranging conditions the environment or habitat condition can alter reproductive fitness, while in captivity (i.e., zoos), enclosure characteristics and enrichments can play an important role in influencing female reproductive success [45]. In accordance with earlier studies from free-ranging animals, we found that under captive conditions, enclosures that contained larger numbers of trees promoted better physiological function among red pandas.

Tree density was the only predictor explaining $47 \%$ of the variation in fPM concentration, again suggesting a more natural habitat in captive conditions is more conducive to breeding activity. The study also showed that fPM concentrations decreased with fGCM concentrations, supporting a potential negative effect on reproduction as has been shown previously [46].

The higher fAM concentrations with frequency of feeding and lower concentrations with higher visitor numbers, as well as among older individuals are in line with relationships between diet and reproductive hormones in captive animals shown by earlier studies. For example, in the giant panda, urinary testosterone was found to be sensitive to flavonoid content, with increasing flavonoids being associated with increased testosterone in males due to intake of phytoestrogens from bamboo [47]. Phytoestrogens are important component closely associated with giant panda reproduction [47]. Though flavonoids are present in minor amounts in bamboo, frequent consumption of bamboo may retain substantial amount in giant pandas to influence reproduction [48,49]. In Japan, a questionnaire survey study in multiple zoos indicated that frequency of bamboo feeding affects reproductive success in captive red pandas [50]. Similarly, our study confirmed that more frequent feeding was associated with increased fAM concentrations and hence might be contributing to reproductive fitness in male red pandas.

That fAM concentration decreased with age in captive male red pandas was not unexpected. Reproductive fitness decreases with age in males and females of many species [51,52]. In giant pandas, fAM concentrations were shown to be age-dependent and increased from 3-7-years of age, most notably between 3-4 years, and then decreased with increasing age [53]. The present study showed lower fAM concentrations 
with increased visitor numbers, which also was not surprising. In a review by Davy [54], negative impacts of visitors on welfare have been observed in captive primates, such as chimpanzees, lion tailed macaques, tamarins, and lemurs. Hediger and Reade [55] in their book "Man and Animal in the Zoo" described how visitor behaviour, for example shouting, teasing, and vandalism can adversely affect captive animals, including reproduction, especially if they cannot retreat from visitors. In spider monkeys, visitor presence increased hypothalamic-pituitary-adrenal activity, which then was associated with reduced reproductive fitness [25].

\section{Conclusions}

Although results are preliminary due to low sample numbers, our study is the first to demonstrate relationships between adrenal and reproductive hormones and several environment and biological factors in pandas housed in a zoo setting. There were negative effects of fGCM on fPM concentration among female red panda in captivity, while fGCM concentrations increased with visitor number, and decreased with frequency of feeding and enclosure area. Female reproductive hormone (fPM) concentrations increased with tree density while male hormones (fAM) increased significantly with frequency of feedings, but decreased with visitor numbers and animal age. The study also demonstrated an inverse relationship between fGCM and fPM, indicating a potential negative influence of stress on reproduction in captive red pandas. Although, a longer-term study is needed to better understand relationships between adrenal and reproductive hormones in captive red pandas, the study suggests that regulating visitors, increasing feeding frequency, and maintaining larger enclosure areas are important factors in zoo management to reduce stress and support better reproductive fitness in this species.

\section{Supplementary Materials:}

Author Contributions: “Conceptualization: N.B., A.S.K. and J.B.; methodology: G.U. and V.K.; software: A.S.K.; validation: G.U. and V.K.; formal lab analysis: A.S.K. and V.K.; investigation: A.S.K.; resources: G.U.; data curation and analysis: N.B. and A.S.K.; writing-original draft preparation: A.S.K. and N.B.; writing-review and editing: N.B., G.U., V.K. and J.B.; visualization: J.B.; supervision: N.B.; project administration: N.B.; funding acquisition: no funding. All authors have read and agreed to the published version of the manuscript." Please turn to the CRediT taxonomy for the term explanation. Authorship must be limited to those who have contributed substantially to the work reported.

Funding: This research received no external funding

Institutional Review Board Statement: “Ethical review and approval were waived for this study, due to the non-invasive approach that involved collection of fecal samples only.

Data Availability Statement: All necessary data supporting reported results are included as part of the manuscript.

Acknowledgments: We are grateful to directors, biologists and veterinarians of all the three zoos to permit and support the study. We sincerely thank all the zookeepers and other zoo staff for helping to carry out the study and provide the ground support in all possible manners.

Conflicts of Interest: The authors declare no conflict of interest.

\section{References}

1. Choudhury, A. An overview of the status and conservation of the red panda Ailurus fulgens in India, with reference to its global status. Oryx 2001, 35, 250-259, doi:10.1046/j.1365-3008.2001.00181.x.

2. Glatston, A.; Wei, F.; Zaw, T. and; Sherpa, A. Ailurus fulgens, red panda assessment. Int. Union Conserv. Nat. 2015, 8235, doi:http://dx.doi.org/10.2305/IUCN.UK.2015- 4.rlts.t714A45195924.en. 
3. Glatston, A.R.; Roberts, M. The current status and future prospects of the red panda (Ailurus fulgens) studbook population. Zoo Biol. 1988, 7, 47-59, doi:10.1002/zoo.1430070106.

4. Wei, F.; Feng, Z.; Wang, Z.; Hu, J. Current distribution, status and conservation of wild red pandas Ailurus fulgens in China. Biol. Conserv. 1999, 89, 285-291, doi:10.1016/S0006-3207(98)00156-6.

5. Eriksson, P.; Zidar, J.; White, D.; Westander, J.; Andersson, M. Current husbandry of red pandas (Ailurus fulgens) in zoos. Zoo Biol. 2010, 29, 732-740, doi:10.1002/zoo.20323.

6. Roka, B.; Chand, P.; Rai, U.; Chhetri, D.R.; Naidu, P.; Zoological, H.; Bengal, W. Study of red panda (Ailurus fulgens fulgens) in ex situ facility for conservation breeding at Padmaja Naidu Himalayan Zoological Park , Darjeeling. International Journal of Zoology Studies. 2018, 3, 184-187.

7. MacDonald, E.A.; Northrop, L.E.; Czekala, N.M. Pregnancy detection from fecal progestin concentrations in the red panda (Ailures fulgens fulgens). Zoo Biol. 2005, 24, 419-429, doi:10.1002/zoo.20059.

8. Princée, F.P.G.; Glatston, A.R. Influence of climate on the survivorship of neonatal red pandas in captivity. Zoo Biol. 2016, 35, 104-110, doi:10.1002/zoo.21266.

9. Morgan, K.N.; Tromborg, C.T. Sources of stress in captivity. Appl. Anim. Behav. Sci. 2007, 102, 262-302, doi:10.1016/j.applanim.2006.05.032.

10. Moberg, G.P. Influence of stress on reproduction: measure of well-being. Anim. Stress 1985, 245-267, doi:10.1007/978-1-4614-7544-6_14.

11. Marinath, L.; Vaz, J.; Kumar, D.; Thiyagesan, K.; Baskaran, N. Drivers of stereotypic behaviour and physiological stress among captive jungle cat (Felis chaus Schreber, 1777) in India. Physiol. Behav. 2019, 210, 112651, doi:10.1016/j.physbeh.2019.112651.

12. Vaz, J.; Narayan, E.J.; Kumar, R.D.; Thenmozhi, K.; Thiyagesan, K.; Baskaran, N. Prevalence and determinants of stereotypic behaviours and physiological stress among tigers and leopards in Indian zoos. PLoS One 2017, 12, 1-27, doi:10.1371/journal.pone.0174711.

13. Chamove, A.S.; Hosey, G.R.; Schaetzel, P. Visitors excite primates in zoos. Zoo Biol. 1988, 7, 359-369, doi:10.1002/zoo.1430070407.

14. Gittleman, J.L. Are the pandas successful specialists or evolutionary failures? Bioscience 1994, 44, 456-464, doi:10.2307/1312297.

15. Delaski, K.M.; Ramsay, E.; Gamble, K.C. Retrospective analysis of mortality in the north american captive red panda (Ailurus fulgens) population, 1992-2012. J. zoo Wildl. Med. Off. Publ. Am. Assoc. Zoo Vet. 2015, 46, 779-788, doi:10.1638/2014-0166.1.

16. Wei, F.; Lü, X.; Li, C.; Li, M.; Ren, B.; Hu, J. Influences of mating groups on the reproductive success of the southern Sichuan red panda (Ailurus fulgens styani). Zoo Biol. 2005, 24, 169-176.

17. Curry, E.; Browning, L.J.; Reinhart, P.; Roth, T.L. Integrating trans-abdominal ultrasonography with fecal steroid metabolite monitoring to accurately diagnose pregnancy and predict the timing of parturition in the red panda (Ailurus fulgens styani). Zoo Biol. 2017, 36, 193-200, doi:https://doi.org/10.1002/zoo.21358.

18. Raja, N.; Budithi, B.; Kumar, V.; Kumar, S. Non-invasive monitoring of reproductive and stress hormones in the endangered red panda (Ailurus fulgens fulgens). Anim. Reprod. Sci. 2016, 172, 173-181, doi:10.1016/j.anireprosci.2016.07.016.

19. Terio, K.A.; Brown, J.L.; Moreland, R.; Munson, L. Comparison of different drying and storage methods on quantifiable concentrations of fecal steroids in the cheetah. Zoo Biol. 2002, 21, 215-222, doi:10.1002/zoo.10036.

20. Brown, J.L.; Wasser, S.K.; Wildt, D.E.; Graham, L.H. Comparative aspects of steroid hormone metabolism and 
ovarian activity in felids, measured noninvasively in feces1. Biol. Reprod. 1994, 51, 776-786, doi:10.1095/biolreprod51.4.776.

21. Umapathy, G.; Kumar, V.; Wasimuddin; Kabra, M.; Shivaji, S. Detection of pregnancy and fertility status in big

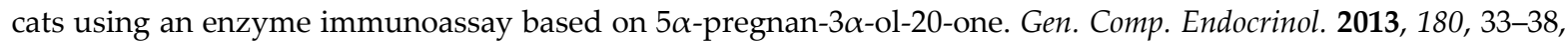
doi:10.1016/j.ygcen.2012.10.009.

22. Kumar, V.; Palugulla Reddy, V.; Kokkiligadda, A.; Shivaji, S.; Umapathy, G. Non-invasive assessment of reproductive status and stress in captive Asian elephants in three south Indian zoos. Gen. Comp. Endocrinol. 2014, 201, 37-44, doi:10.1016/j.ygcen.2014.03.024.

23. Mithileshwari, C.; Srivastava, T.; Kumar, V. Theriogenology Non-invasive assessment of fecal progestagens and pregnancy detection in Himalayan musk deer (Moschus chrysogaster). Theriogenology 2016, 85, 216-223, doi:10.1016/j.theriogenology.2015.09.009.

24. Goodenough, A.E.; Hart, A.G.; Stafford, R. Regression with empirical variable selection: Description of a new method and application to ecological datasets. PLoS One 2012, 7, doi:10.1371/journal.pone.0034338.

25. Davis, N.; Schaffner, C.M.; Smith, T.E. Evidence that zoo visitors influence HPA activity in spider monkeys (Ateles geoffroyii rufiventris). Appl. Anim. Behav. Sci. 2005, 90, 131-141, doi:10.1016/j.applanim.2004.08.020.

26. Sherwen, S.L.; Hemsworth, P.H. The visitor effect on zoo animals: implications and opportunities for zoo animal welfare. animals 2019, 1-27.

27. Pifarré, M.; Valdez, R.; González-Rebeles, C.; Vázquez, C.; Romano, M.; Galindo, F. The effect of zoo visitors on the behaviour and faecal cortisol of the Mexican wolf (Canis lupus baileyi). Appl. Anim. Behav. Sci. 2012, 136, 57-62, doi:10.1016/j.applanim.2011.11.015.

28. Rajagopal, T.; Archunan, G.; Sekar, M. Impact of zoo visitors on the fecal cortisol levels and behavior of an endangered species: Indian Blackbuck (Antelope cervicapra L.). J. Appl. Anim. Welf. Sci. 2011, 14, 18-32, doi:10.1080/10888705.2011.527598.

29. Gittleman, J.L. Behavioral energetics of lactation in a herbivorous carnivore, the red panda (Ailurus fulgens). Ethology 1988, 79, 13-24, doi:10.1111/j.1439-0310.1988.tb00696.x.

30. Rees, P.A. Activity budgets and the relationship between feeding and stereotypic behaviors in Asian elephants (Elephas maximus) in a Zoo. Zoo Biol. 2009, 28, 79-97, doi:10.1002/zoo.20200.

31. Varadharajan, V.; Krishnamoorthy, T.; Nagarajan, B. Prevalence of stereotypies and its possible causes among captive Asian elephants (Elephas maximus) in Tamil Nadu, India. Appl. Anim. Behav. Sci. 2015, 174, 137-146, doi:10.1016/j.applanim.2015.10.006.

32. Foley, C.A.H.; Papageorge, S.; Wasser, S.K. Noninvasive stress and reproductive measures of social and ecological pressures in free-ranging African elephants. Conserv. Biol. 2001, 15, 1134-1142, doi:10.1046/j.1523-1739.2001.0150041134.x.

33. Muller, M.N.; Wrangham, R.W. Dominance, cortisol and stress in wild chimpanzees (Pan troglodytes schweinfurthii). 2004, 332-340, doi:10.1007/s00265-003-0713-1.

34. Clutton-Brock, T.H. Mammalian mating systems. Proc. R. Soc. London. Ser. B, Biol. Sci. 1989, 236, 339-372, doi:10.1098/rspb.1989.0027.

35. Clubb, R.; Mason, G. Captivity effects on wide-ranging carnivores. Nature 2003, 425, 473-474, doi:10.1038/425473a.

36. Bista, D.; Shrestha, S.; Sherpa, P.; Thapa, G.J.; Kokh, M.; Lama, S.T.; Khanal, K.; Thapa, A.; Jnawali, S.R. Distribution and habitat use of red panda in the Chitwan-Annapurna Landscape of Nepal. PLoS One 2017, 12, 
1-16, doi:10.1371/journal.pone.0178797.

37. Panthi, S.; Khanal, G.; Acharya, K.P.; Aryal, A.; Srivathsa, A. Large anthropogenic impacts on a charismatic small carnivore: Insights from distribution surveys of red panda Ailurusfulgens in Nepal. PLoS One 2017, 12, 1-14, doi:10.1371/journal.pone.0180978.

38. Little, K.A.; Sommer, V. Change of enclosure in langur monkeys: Implications for the evaluation of environmental enrichment. Zoo Biol. 2002, 21, 549-559, doi:10.1002/zoo.10058.

39. Sade, C.; Burchett, S. Visitor effects on zoo animals. Plymouth Student Sci. 2013, 6, 423-433.

40. Vaz, J.; Narayan, E.J.; Kumar, R.D.; Thenmozhi, K.; Thiyagesan, K.; Baskaran, N. Prevalence and determinants of stereotypic behaviours and physiological stress among tigers and leopards in Indian zoos. PLoS One 2017, 12, 1-27, doi:10.1371/journal.pone.0174711.

41. Quirke, T.; O'Riordan, R.M.; Zuur, A. Factors influencing the prevalence of stereotypical behaviour in captive cheetahs (Acinonyx jubatus). Appl. Anim. Behav. Sci. 2012, 142, 189-197, doi:10.1016/J.applanim.2012.09.007.

42. Roberts, B.M.S.; Gittleman, J.L. Ailurus fulgens. Mamm. Species 1984, 222, 1-8.

43. Cook, R.C.; Murray, D.L.; Cook, J.G.; Zager, P.; Monfort, S.L. Nutritional influences on breeding dynamics in elk. Can. J. Zool. 2001, 853, 845-853, doi:10.1139/cjz-79-5-845.

44. Alfaro, J.L.; Valdez, R.A.; Romano, M.C. Stress in Yucatan spider monkeys: effects of environmental conditions on fecal cortisol levels in wild and captive populations. Anim. Conserv. 2009, 12, 496-502, doi:10.1111/j.1469-1795.2009.00280.x.

45. Shepherdson, D.; Carlstead, K. Understanding the relationship between environment and reproduction in captive animals: The role of environmental enrichment. Zoo Biol. 1994, 13, 447-458.

46. Scarlata, C.D.; Elias, B.A.; Godwin, J.R.; Powell, R.A.; Shepherdson, D.; Shipley, L.A.; Brown, J.L. Relationship between fecal hormone concentrations and reproductive success in captive pygmy rabbits (Brachylagus idahoensis) . J. Mammal. 2012, 93, 759-770, doi:10.1644/11-mamm-a-223.1.

47. Liu, H.; Zhang, C.; Liu, Y.; Duan, H. Total flavonoid contents in bamboo diets and reproductive hormones in captive pandas: exploring the potential effects on the female giant panda (Ailuropoda melanoleuca). Conserv. Physiol. 2019, 7, 1-10, doi:10.1093/conphys/coy068.

48. Gittleman, J.L. The Giant Pandas of Wolong. George B. Schaller, Hu Jinchu, Pan Wenshi, Zhu Jing . Q. Rev. Biol. 1985, 60, 524-525, doi:10.1086/414647.

49. Keski-Saari, S.; Ossipov, V.; Julkunen-Tiitto, R.; Jia, J.; Danell, K.; Veteli, T.; Guiquan, Z.; Yaowu, X.; Niemelä, P. Phenolics from the culms of five bamboo species in the Tangjiahe and Wolong Giant Panda Reserves, Sichuan, China. Biochem. Syst. Ecol. 2008, 36, 758-765, doi:10.1016/j.bse.2008.08.003.

50. Tanaka, A.; Ogura, T. Current husbandry situation of red pandas in Japan. Zoo Biol. 2018, 37, 107-114, doi:10.1002/zoo.21407.

51. Jones, O.R.; Scheuerlein, A.; Salguero-Gómez, R.; Camarda, C.G.; Schaible, R.; Casper, B.B.; Dahlgren, J.P.; Ehrlén, J.; García, M.B.; Menges, E.S.; et al. Diversity of ageing across the tree of life. Nature 2014, 505, 169-173, doi:10.1038/nature12789.

52. Lemaître, J.F.; Gaillard, J.M. Reproductive senescence: new perspectives in the wild. Biol. Rev. 2017, 92, 2182-2199, doi:10.1111/brv.12328.

53. Kersey, D.C.; Wildt, D.E.; Brown, J.L.; Huang, Y.; Snyder, R.J.; Monfort, S.L. Parallel and seasonal changes in gonadal and adrenal hormones in male giant pandas (Ailuropoda melanoleuca). J. Mammal. 2010, 91, 1496-1507, doi:10.1644/09-MAMM-A-404.1. 
54. Davey, G. Visitors' effects on the welfare of animals in the zoo: A review. J. Appl. Anim. Welf. Sci. 2007, 10, 169-183, doi:10.1080/10888700701313595.

55. Hediger Reade, Winwood., H. Man and animal in the zoo : zoo biology. London : Routledge and Kegan Paul. 1969; ISBN 07100636879780710063687. 